\title{
Pressure Sensing Using Micromachined Asymmetric Integrated Vertical Coupler
}

\author{
Isa Kiyat, Coskun Kocabas and Atilla Aydinli \\ Department of Physics \\ Bilkent University \\ 06800, Ankara, Turkey \\ Email: kiyat@fen.bilkent.edu.tr
}

\begin{abstract}
Analysis of a novel pressure sensor based on a SOI asymmetric vertical coupler is presented. The integrated optical component is a coupler composed of a single mode low index waveguide and a thin silicon slab.
\end{abstract}

\section{INTRODUCTION}

The pressure sensing by integrated optical means is always attracted much attention, since these sensors can be controlled remotely by optical fibres and they are immune to electromagnetic radiation. Many complicated devices including those using a Mach Zehnder interferometer (MZI) and a ring resonator have been designed and fabricated so far [1,2].

Here, we utilize wavelength selective coupling between a low index single mode (SM) waveguide and a multimode high index semiconductor slab waveguide. The change in refractive index of the diaphragm under pressure alters the transmission spectrum which can be used to read out the applied pressure. Finite element method (FEM) is used to predict the behavior of the membrane under pressure while the optical response of the sensor is analyzed with well known mode calculations and the coupled mode theory. The results are confirmed by $2 \mathrm{D}$ beam propagation method (BPM) simulations.

\section{SENSOR DESIGN AND OPERATION}

The sensor design allows it to be fabricated on a SOI wafer with a few micrometer top silicon thickness and a buried oxide thickness large enough for optical confinement which we choose as 1.5 and $1 \mu \mathrm{m}$ respectively. A $3 \mu \mathrm{m}$ wide and $3 \mu \mathrm{m}$ high, benzocyclobutane (BCB) SM rib waveguide is placed on the SOI substrate after a $2 \mu \mathrm{m} \mathrm{SiO}{ }_{2}$ deposition on top of the Si layer. The structure can be seen in Fig. 1.

The refractive index of BCB polymer is taken as 1.5339 . Other low index materials, namely doped-SiO2, $\mathrm{SiN}$ and SiON, can also be used as the low index SM waveguide. It has been shown that such devices are highly wavelength selective[3]. In Fig. 2, effective indices of the BCB SM waveguide and the highest order mode of the $\mathrm{Si}$ slab waveguides with oxide and air substrates are given as a function of free space wavelength. It is clearly seen that, SM waveguide is phase matched with slab waveguides of different lower claddings at quite different and well separated wavelengths which we label as $\lambda_{a i r}$ and $\lambda_{\text {oxide }}$. The sensor has a diaphragm at the backside of the SOI wafer which can be defined by anisotropic $\mathrm{KOH}$ etch of silicon and followed by a HF etch of the buried oxide. (a)

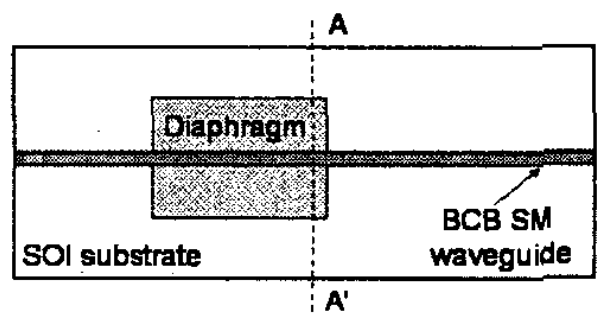

(b)



Fig. 1. Schematic top (a) and AA' cross sectional (b) views of the proposed SOI asymmetric integrated vertical coupler pressure sensor

Thus, the lower cladding of the Si slab waveguide becomes air at the position of the diaphragm opening. The structure is a vertical coupler at these selected wavelengths with different coupling lengths which are calculated

It can be shown that the transmitted power in a waveguide coupler is a function of the phase mismatch between two waveguides and it is [4]

$$
T=\left(\frac{\pi}{2}\right)^{2} \operatorname{sinc}^{2}\left\{\frac{1}{2}\left[1+\left(\frac{\Delta \beta L_{c}}{\pi}\right)^{2}\right]^{1 / 2}\right\}
$$

where $\Delta \beta$ is the propagation constant difference or phase mismatch between waveguide modes, $L_{c}$ is the coupling length and $\operatorname{sinc}(x)=\sin (\pi x) /(\pi x)$. Phase mismatch can be 


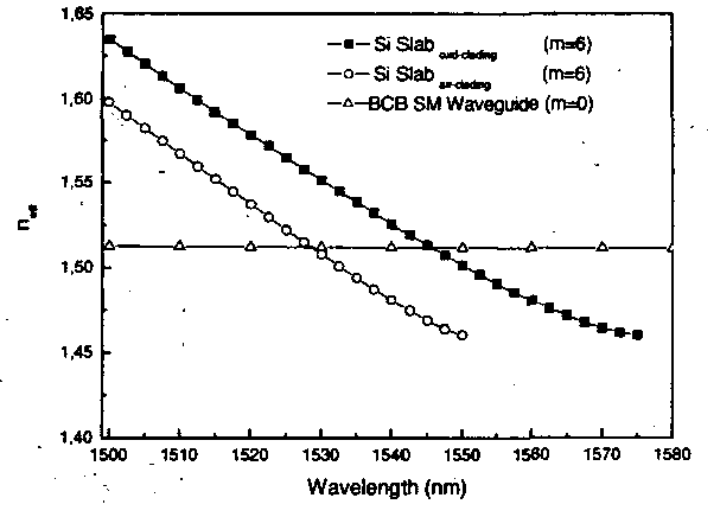

Fig. 2. Effective TE refractive index change with free space wavelength for highest order mode ( $m=6$ ) of $\mathrm{Si}$ slab with $\mathrm{SiO}_{2}$ and air as lower cladding and SM BCB waveguide

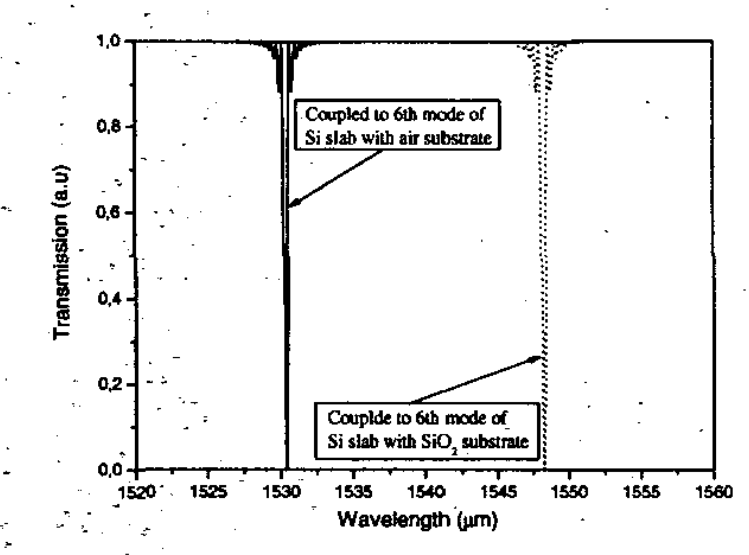

Fig. 3. Transmission spectrum of the SM waveguide integrated on the silicon diaphragm

written in terms of effective index difference and free space wavelength as $\Delta \beta=\Delta \mathrm{n} 2 \pi / \lambda$. It is seen in Fig. 2. effective index of SM BCB waveguide is nearly constant while that of the 6th mode of Si slab is linear around coupling wavelengths, $\lambda_{\text {air }}$ and $\lambda_{\text {oxide. }}$. Thus this approximation can be used to analyze the spectral properties of transmitted power which is one minus transferred power that is 1-T. This spectral response can be seen in Fig. 3 for the calculated coupling lengths of about $1.7 \mathrm{~mm}$. It is seen that the bandwidth gets narrower as is the coupling length and asymmetry between waveguides increases. Therefore, narrower bandwidths results in higher sensitivity which can be achieved by highly asymmetric and well separated (for larger $L_{c}$ ) waveguides. The length of the diaphragm is defined by the coupling length of asymmetric vertical coupler with air as lower cladding for Si slab waveguide. The width is chosen a so as to enhance the induced stress on the silicon slab.

\section{SENSOR SENSITIVITY}

When the input laser wavelength is chosen near $\lambda_{\text {air }}$, any change in the refractive index of the system can be observed

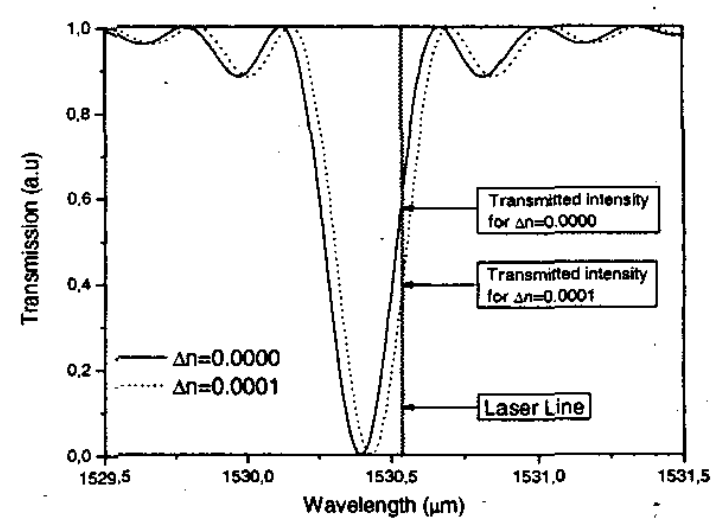

Fig. 4. The change in transmission spectrum of the SM waveguide is clearly seen when refractive index of the $\mathrm{Si}$ slab changes under applied pressure.

in transmitted intensity. In Fig. 4, a change of 0.0001 in Si refractive index results in a net shift of the transmission dip position ( $\left.\lambda_{\text {air }}\right)$ and transmitted intensity decreases to 70 of its original value. So, it is seen that as the transmission dip gets narrower the intensity drop gets larger. Our design is capable of sensing even smaller refractive index changes therefore even smaller pressures.

The choice of a polymer as SM waveguide is due to the fact that its refractive index change under pressure is very small compared to silicon, and is ignored in analysis. The refractive index change of silicon slab with applied pressure is calculated with .FEM and it is found that there is a linear relationship between index change and applied pressure. The net change in silicon index is calculated to be 0.0001 for a pressure of $25 \mathrm{kPa}$, using elosto-optic coefficients for silicon [5]. Thus, the sensitivity of transmitted intensity is calculated by $\Delta \mathrm{V} / \mathrm{P}$ and is found to be about $0.01 \mathrm{kPa}^{-1}$, where $\Delta \mathrm{I}$ and $\Delta \mathrm{P}$ are change in transmitted intensity and pressure applied, respectively.

\section{CONCLUSION}

A novel pressure sensor based on a SOI asymmetric integrated vertical coupler is designed and analyzed. The proposed device is both easy to design and fabricate, compared to previous sensor designs. It uses well established silicon micromachining technology applied to SOI substrates. Newly developed SOI wafers with highly unifôrm thicknesses are well suited for this device. The sensor comes out to be very sensitive due to dependence of transmission of the asymmetric coupler on refractive index of $\mathrm{Si}$ slab and can be used for but not limited to the low pressure regime.

\section{REFERENCES}

[1] Gregory N. T. B. et al, IEEE Photon. Technol. Lett., 6(1994), 671. :

[2] Porte H., et al, J. Lightwave Technol., 17 (1999), 229.

[3] Pezeshki B. et al, Appl. Phys. Lett., 68 (1996), 741.

[4] Saleh E. A. B. et al, Fundamentals of Photonics, (John Wiley \& Sons, NY, 1991), 267.

15] Weber, M.J. Handbook of Laser Science and Technology, (CRC, Boca, Fl, 1986), 325 . 